\section{NOTAS DE CLASE}

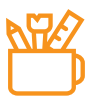

\title{
LAS DECISIONES
}

FINANCIERAS

Edder Parody Camargo

Universidad Cooperativa de Colombia

Seccional Santa Marta

Documentos de docencia | Course Work

Coursework.ucc.edu.co

$N^{0} 6$, julio de 2018

Doi: https://doi.org/10.16925/greylit.2406

\section{NOTA LEGAL}

El presente documento de trabajo ha sido incluido dentro de nuestro repositorio de literatura gris por solicitud del autor, con fines informativos, educativos o académicos. Asimismo, los argumentos, datos y análisis

incluidos en el texto son responsabilidad absoluta del autor y no representan la opinión del Fondo Editorial o de la Universidad.

\section{DISCLAIMER}

This coursework paper has been uploaded to our grey literature repository due to the request of the author. This document should be used for informational, educational or academic purposes only. Arguments, data and analysis included in this document represent authors' opinion not the Press or the University 


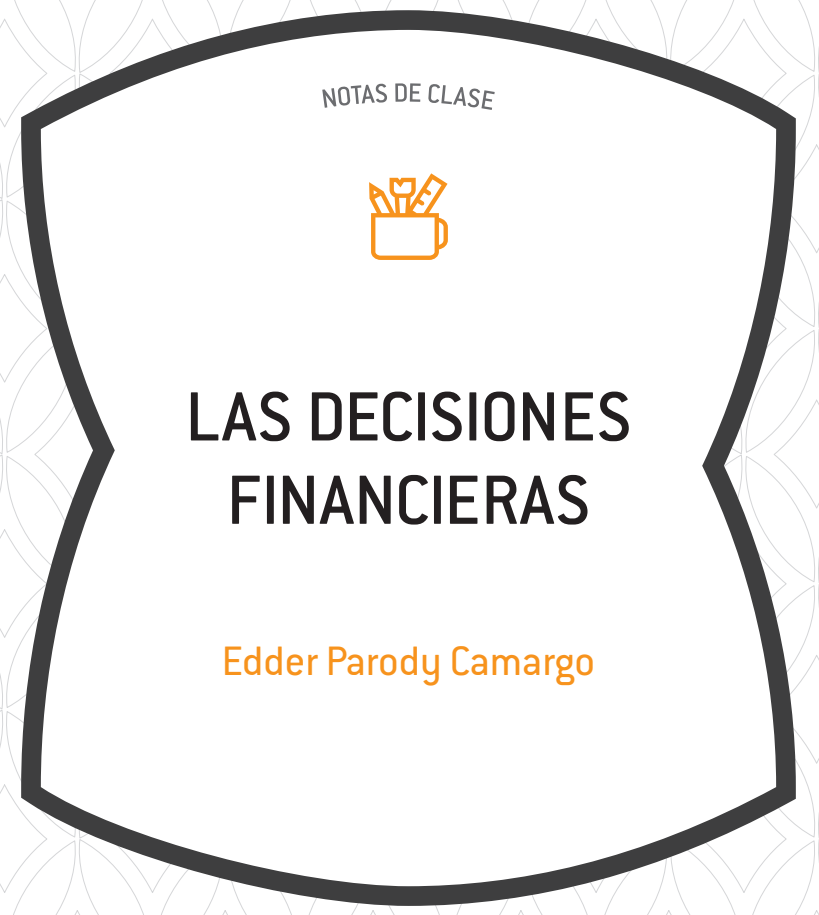




\section{ACERCA DEL AUTOR}

Edder Parody-Camargo, magister en finanzas, profesor asistente del Programa de Administración de Empresas, Universidad Cooperativa de Colombia, seccional Santa Marta, Colombia.

Correo electrónico:

edder.parodyc@campusucc.edu.co

\section{Cómo CITAR ESTE DOCUMENTO}

Parody-Camargo, E. (2018). Las decisiones financieras. (Documento de docencia $N^{\circ}$ 6). Bogotá: Ediciones Universidad Cooperativa de Colombia. Doi: https:// doi.org/10.16925/greylit.2406

Este documento puede ser consultado, descargado o reproducido desde nuestro repositorio de documentos de trabajo (http://coursework. ucc.edu.co) para uso de sus contenidos, bajo la licencia de Creative Commons Reconocimiento-NoComercial-SinObraDerivada 4.0 Internacional. http://creativecommons.org/licenses/by-nc-nd/4.0/

(c) (1) (3) 


\section{TABLA DE CONTENIDO}

INTRODUCCIÓN

UNIDAD 1. LAS TASAS DE INTERÉS

$\begin{array}{ll}\text { Prefacio } & 7\end{array}$

$\begin{array}{ll}\text { Desarrollo } & 7\end{array}$

Variación del poder adquisitivo $\quad 7$

Tasas de interés 8

Elementos que integran las tasas de interés 8

Diferencia entre interés nominal e interés efectivo 9

CASOS EN TASAS DE INTERÉS 10

Caso 1: de efectiva a efectiva 10

Caso 2: de efectiva (periódica) a nominal con el mismo periodo de capitalización $\quad 10$

Caso 3: de efectiva (periódica) a nominal con diferente periodo de capitalización 10

Caso 4: de nominal a efectiva (periódica) con igual periodo de capitalización $\quad 11$

Caso 5: de nominal a efectiva (periódica) con diferente periodo de capitalización $\quad 11$

Caso 6: de periódica vencida a periódica anticipada 12

Caso 7: de periódica anticipada a periódica vencida 12

Recomendaciones para trabajar las tasas de interés 12

¡Atención! 13

$\begin{array}{ll}\text { TASA REAL O DEFLACTADA } & 14\end{array}$

$\begin{array}{ll}\text { Apartado final } & 15\end{array}$

$\begin{array}{ll}\text { EJERCICIOS PARA PRACTICAR } & 16\end{array}$

$\begin{array}{ll}\text { REFERENCIAS } & 18\end{array}$

ÍNDICE FIGURAS Y TABLAS

Figura 1. Esquema de conversión de tasas $\quad 9$

Figura 2. Función Tasa.Nominal 10

Figura 3. Función InT.EFECTIVo 11

Tabla 1. Diferencia entre interés nominal e interés efectivo 9 


\section{LAS DECISIONES FINANCIERAS}

Edder Parody Camargo

\section{Resumen}

El objetivo del presente documento es el desarrollo de una guía práctica que permita al estudiante generar bases para la toma de decisiones acertadas, este se encuentra estructurado en una sección, en esta se mostrará la parte teórica de las tasas de interés, su representación literal, las fórmulas para desarrollarlas y un grupo de ejercicios para la práctica. Se utiliza las fórmulas financieras de Microsoft Excel, atendiendo las competencias del uso de los sistemas de información ante un mundo globalizado. En conclusión, se pretende que el estudiante alcance la unidad de competencia propuesta en el plan de curso toma de decisiones financieras que se enfoca en la toma de decisiones financieras de inversión y de financiación para la sustentabilidad de la organización, mediante el uso de las matemáticas financieras.

Palabras clave: decisiones, financiación, inversión, sustentabilidad, tasas. 


\section{INTRODUCCIÓN}

El propósito de este documento es brindar al estudiante herramientas que le permitan identificar y seleccionar alternativas de inversión y financiación, proceso que lleva implícito la toma de decisiones, aplicando las matemáticas financieras. Los gerentes constantemente se encuentran en situaciones, ya sea de déficit o de superávit, en las cuales necesitarán recursos para financiar sus actividades, por ello, deben recurrir al mercado financiero, que cobra un margen por la intermediación que realiza, esto se denomina tasa de interés, entonces, se tiene una gama de opciones entre las cuales elegir, cabe preguntarse ¿cuál es la entidad financiera que se adapta a mis condiciones? por ello la importancia de conocer la temática a abordar.
Así mismo, los agentes superavitarios ${ }^{1}$ tienen exceso de recursos, por lo tanto, deben colocar el dinero en inversiones que generen valor, los diferentes mercados como son, el de capitales, y el monetario, entre otros. Al colocar el dinero en inversiones, generan una renta o una perdida, entonces ¿en cuáles invertir? Igualmente, algunas variables de la economía afectan el valor del dinero en el tiempo, por ello es necesario conocerlas y saber su incidencia en ese valor. Esto puede hacerse con el uso de la calculadora o con el software Excel de Microsoft. Este documento muestra el elemento teórico de las tasas de interés, su representación literal, las fórmulas para desarrollarlas y un grupo de ejercicios para la práctica. 


\section{UNIDAD 1. LAS TASAS DE INTERÉS}

\section{Prefacio}

Al terminar esta unidad, el estudiante tendrá algunas nociones que le servirán de base para la toma de decisiones financieras de inversión y de financiación para la sustentabilidad² de la organización. También, habrá estudiado variables como el riesgo, inflación, y su efecto en las tasas de interés, para ello debe tener conocimientos previos de interpretación de variables económicas, en el uso de Excel.

\section{DESARROLLO}

Las empresas, constantemente, se ven sumergidas en la necesidad de financiar sus operaciones, sus inversiones, estos recursos se pueden generar de manera interna o externa, sin embargo, esto tiene implícitamente unos costos, desde el punto de vista del inversor es necesaria una rentabilidad, pero desde la perspectiva del mercado financiero exige un interés. Por lo tanto, el usuario del dinero, activos o servicios debe satisfacer los deseos de utilidad de quien los proporciona (García, 2008). Por lo anterior es preciso señalar que el dinero no es gratis, $y$ que, además, toma diferentes valores a través del tiempo.

El Valor del Dinero en el Tiempo, en adelante VDT, es entendido como el valor que puede tomar el dinero en los diferentes periodos de tiempo, es decir, que el poder adquisitivo de una persona con una cantidad de dinero puede aumentar o disminuir. Para ampliar la explicación, el siguiente ejemplo:

\footnotetext{
2 La sustentabilidad no meramente económica sino también vista desde otras dos aristas, como son la biocontabilidad y la sociocontabilidad definidas como disciplinas científicas encargadas de evaluar la gestión de la organización en el control de la riqueza ambiental y social, en función de la importancia de la naturaleza y la sociedad, en el marco de una fundamentación biocéntrica - o ecocéntrica - de la contabilidad, apartada de los cálculos exclusivamente rentísticos y financieros (Soto y Serna, 2015).
}

\section{Variación del poder adquisitivo}

Matías tiene 500 pesos, con eso quiere comprar una galleta, que tiene un precio de 500 pesos, pero decide no comprar la galleta y guarda su dinero, una semana después decide comprarla, pero resulta que la variable económica llamada inflación subió de 5\% a 5.5\%, y generó un efecto sobre el precio de los productos, aumentando el precio de la galleta en $\$ 550$, lo que quiere decir, que el joven, con la misma cantidad de dinero, no podrá comprar su galleta, es un claro ejemplo de pérdida de poder adquisitivo, dado que ahora solo puede comprar 0.9090 (500/550) de la galleta, es decir, con la misma suma compra menos cantidad del producto.

Entonces, se puede afirmar, como lo menciona Meza (2008), que hay unas variables que hacen que el dinero gane o pierda valor a través del tiempo, para ello, menciona que el costo de oportunidad es aquello que se sacrifica cuando tomamos una decisión, por ejemplo, una persona puede optar por descansar en vez de trabajar, no se tiene que pagar por ello, pero en realidad tiene un costo, que llaman costo de oportunidad. En cuanto a la inflación, el Banco de la Republica (2017) define el fenómeno de la inflación como un aumento sustancial, persistente y sostenido del nivel general de precios a través del tiempo, en términos prácticos, hace que el dinero día a día pierda poder adquisitivo como se explicó anteriormente. Otra variable es el riesgo, toda inversión lleva implícito un riesgo, dado que siempre estará presente la incertidumbre, al no conocer con certeza si la inversión que se está realizando generará el retorno esperado, por consiguiente, el riesgo de perder en la inversión influye en el costo del dinero.

En los conceptos anteriores se pudo constatar que el dinero tiene un valor en el tiempo, ese valor se manifiesta a través del interés, lo que traduce que el interés es la medida o 
la manifestación del VDT, es decir, el uso del dinero no es gratuito y financieramente se evidencia en la tasa de interés. A continuación, se presentan definiciones de las diferentes tasas utilizadas en el mercado financiero.

\section{Tasas de interés}

Es un indicador que se expresa en forma de porcentaje y se usa para estimar el costo de un crédito o la rentabilidad de los ahorros o inversión. La tasa de interés establece un balance entre el riesgo y la posible ganancia de la utilización de una suma de dinero en una situación y tiempo determinado (ASOBANCARIA, 2015). En el mercado financiero existen dos tipos de tasas, las tasas de interés nominales y las tasas de interés efectivas.

Tasas de interés nominal: la tasa nominal es aquella que pueden ser otorgada por las entidades financieras, pero realmente no alcanzan a medir el costo ni la rentabilidad de una inversión:

Esta tasa se refiere generalmente a una tasa anual, pero con una característica especial: es susceptible de fraccionamiento entre un numero de capitalizaciones al año. Por esta razón, cuando en el enunciado de un problema se dice que la tasa nominal o anual es capitalizable en fracciones de año, hay que dividir dicha tasa nominal sobre el número de veces que capitaliza en el año. (Gomero, 2006, p. 49)

Las tasas nominales se pueden expresar de la siguiente manera:

- $12 \%$ nominal mensual vencida ${ }^{3}$

- $\quad 12 \%$ anual con capitalización mensual

- $12 \%$ anual con capitalización mensual vencida

3 El número 12 en este caso es un ejemplo dado que puede ser el 1 , 2, 3, 4, 5, 6, 7, 8, 9, 10, 11 u otros.
- $12 \%$ nominal anual mes anticipado

- $\quad 12 \%$ NMV (Nominal Mes Vencido)

- $\quad 12 \%$ NMA (Nominal Mes Anticipado)

- $12 \%$ nominal pagadero mensualmente

- $12 \%$ anual liquidable mensualmente por anticipado

- $12 \%$ anual capitalizable mensualmente

- $\quad 12 \%$ nominal mensual

- $12 \%$ mV (Mensual Vencido)

- $\quad$ 12\% MA (Mensual Anticipado)

Tasas de interés efectivas: La tasa efectiva es aquella a la que se llega cuando se capitalizan los intereses pagados a la misma tasa a la cual se pactó en el negocio al comienzo del periodo (Sabogal, Cayón y Rivera, 2007). Las tasas efectivas pueden expresarse de la siguiente manera:

- $15 \%$ efectivo anual

- $15 \%$ EA

- $\quad 15 \%$ TEA

- $2 \%$ mensual

- $2 \%$ mensual vencido

- $2 \%$ periódica mensual

- $2 \%$ PMV (Periódica Mensual Vencida)

De acuerdo con el ejemplo anterior, la lógica evidencia que las tasas periódicas son menores que las tasas anuales, por lo que se realizan en periodos inferiores a un año.

\section{Elementos que integran las tasas de interés}

Siempre que se trabaje una operación en interés compuesto, se deben tener en cuenta los tres elementos de las tasas de interés:

1. Tipo: se refiere a si es nominal o si es efectiva. 
2. Unidad: está relacionada con el periodo de pago (en el caso de la efectiva) o de capitalización del interés en el caso de la nominal.

3. Condición: representa el momento de cobro del interés, que puede ser al inicio (tasa anticipada) o al final (tasa vencida).

\section{Diferencia entre interés nominal e interés efectivo}

Nótese en la tabla 1 que la diferencia radica en que en las tasas nominales los periodos de plazo y capitalización son diferentes, mientras que los mismos periodos en la efectiva son iguales, por consiguiente, cada vez que se tenga una operación financiera, es necesario trabajar tasas efectivas, es decir si tiene una tasa nominal esta debe ser transformada en efectiva.

Tabla 1

Diferencia entre interés nominal e interés efectivo

NOMINAL

\begin{tabular}{|c|c|c|c|}
\hline $15 \%$ & Anual & Capitalizable Mes & Vencido \\
\hline Tasa & Plazo & $\begin{array}{c}\text { Periodo de capitalización } \\
\text { Periodo de liquidación }\end{array}$ & $\begin{array}{c}\text { Momento de pago } \\
\text { o de capitalización }\end{array}$ \\
\hline
\end{tabular}

\begin{tabular}{|c|c|c|c|}
\hline \multicolumn{3}{c}{ EFECTIVA } \\
\hline $15 \%$ & Anual & Capitalizable Anualmente & Vencido \\
\hline Tasa & Plazo & $\begin{array}{c}\text { Periodo de capitalización } \\
\text { Periodo de liquidación }\end{array}$ & $\begin{array}{c}\text { Momento de pago } \\
\text { o de capitalización }\end{array}$ \\
\hline
\end{tabular}

Nota. Elaboración propia con información de Ramírez y Martínez (2010) y ASOBANCARIA (2015).

A continuación, se presenta un esquema gr que nos sirve de guía para la transformación o conversión de tasas nominales a periódicas y efectivas, o viceversa.

Para hacer conversión de periódicas a periódicas (vencidas). Es sencillo, solo debes tener en cuenta el factor $(\mathbf{1}+\mathbf{i})$ y luego remplazar $n$ y $m$ de la siguiente manera.

$\mathrm{n}=$ De dónde vengo, de que periodicidad vengo.

$\mathrm{m}=$ Hacia donde voy, que periodicidad quiero encontrar.

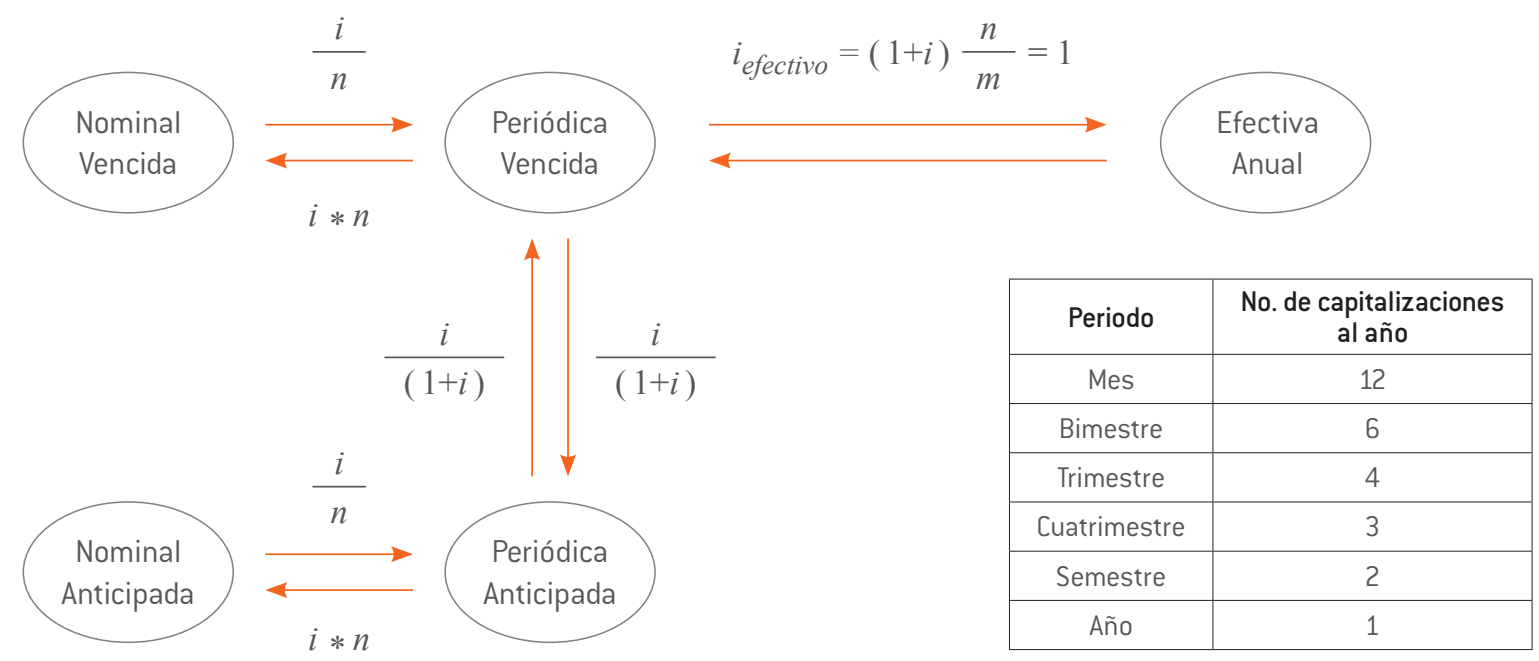

Figura 1. Esquema de conversión de tasas. Elaboración propia con información de Sosa (2006). 


\section{CASOS EN TASAS DE INTERÉS}

\section{CASO 1: DE EFECTIVA A EFECTIVA}

En este caso tenemos una tasa efectiva conocida y se quiere hallar otra tasa efectiva equivalente, por ejemplo, del 2\% mensual, hallar una tasa semestral.

Para este caso es necesario entender que solo se requiere cambiar el periodo, utilizamos la fórmula que aparece en el grafico 1: i $i_{\text {efectivo, }}$ para este caso tenemos lo siguiente:

$$
i_{\text {efectivo }}=\left(1+i_{\text {efectivo }}\right) \frac{n}{m}-1
$$

Tenemos entonces que el desarrollo quedaría de la siguiente manera

$$
\text { interés }_{\text {efectivo }}=(1+2 \%)\left(\frac{12}{2}\right)-1=12.62 \% \text { semestral }
$$

Ahora cabe explicar que el numerador del exponente es 12, lo que significa que la tasa que tenemos es mensual, y en un año hay 12 periodos de capitalización mensual, y en el numerado es 2 , por lo que el periodo que se requiere es semestral, y en un año hay 2 periodos de capitalización semestral.

\section{CASO 2: DE EFECTIVA (PERIÓDICA) A NOMINAL CON EL MISMO PERIODO DE CAPITALIZACIÓN}

Para este caso tenemos una tasa efectiva (periódica) conocida, y se requiere hallar una tasa nominal con el mismo periodo de capitalización, por ejemplo, del 3\% trimestral hallar una tasa nominal trimestre vencido (NTV). Para este caso es necesario entender que se va a llevar a una nominal que tiene el mismo periodo de capitalización, estas nominales son una expresión anual de las tasas periódicas, por consiguiente, solo es necesario multiplicarlas por el número de veces que capitaliza en el año. En el grafico 1 se observa de periódica vencida a nominal vencida, y se desarrolla de la siguiente manera:

$$
i_{\text {efectivo }}=i_{\text {efectivo }} * N^{\circ} \text { de periodos de capitalización }
$$

Para el caso de estudio, los periodos de capitalización trimestral que tiene un año son 4, por tanto, tenemos:

$$
i_{\text {nominal }}=3 \% * 4=12 \% \text { nominal trimestral vencida }
$$

Para el caso de la Efectiva Anual (EA) a una nominal vencida, se puede trabajar con la función financiera en Microsoft Excel, denominada Tasa. Nominal, como a continuación se muestra:

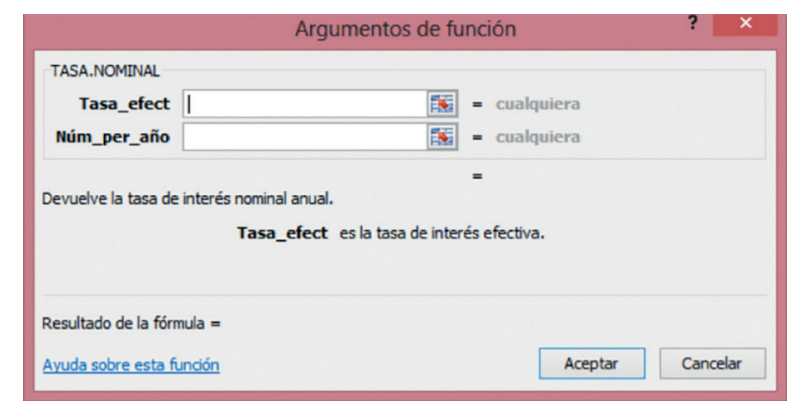

Figura 2. Función Tasa.Nominal. Tomada de Microsoft (2016)

Como se puede observar en la figura 1, los argumentos que pide la función son Tasa_efect y Núm_per_año, el primero es la tasa efectiva conocida, y el segundo es la tasa nominal que se quiere, para el caso de la nominal mensual el número de periodos de capitalización al año es 12, para la trimestral 4 y así sucesivamente.

\section{CASO 3: DE EFECTIVA (PERIÓDICA) A NOMINAL CON DIFERENTE PERIODO DE CAPITALIZACIÓN}

Para este caso tenemos una tasa efectiva (periódica) conocida y se requiere hallar una tasa nominal con diferente periodo de capitalización, por ejemplo, del 3\% trimestral hallar una tasa Nominal Semestre Vencido (NSV).

Para este caso es necesario entender que se va a llevar a una nominal que tiene diferente periodo de capitalización, estas nominales son una expresión anual de las tasas periódicas, 
por consiguiente, se debe primero cambiar la periodicidad, posteriormente, multiplicarla por el número de veces que capitaliza en el año.

Para cambiar la periodicidad solo se puede desarrollar cuando están efectivas y vencidas, para este caso se tiene:

$$
i_{\text {efectivo }}=\left(1+i_{\text {efectivo }}\right) \frac{n}{m}-1
$$

Su desarrollo sería:

$$
\text { interés }_{\text {efectivo }}=(1+3 \%)\left(\frac{4}{2}\right)-1=6.09 \% \text { semestral }
$$

Luego, ya se cambió la periodicidad, ahora solo queda multiplicarla por el número de veces que capitaliza en el año, para el caso de estudio, el número de veces que capitaliza los semestres en el año son 2, quedaría de la siguiente manera:

$$
i_{\text {nominal }}=6.09 \% * 2=12.18 \% \text { nominal semestral vencida }
$$

\section{CASO 4: DE NOMINAL A EFECTIVA (PERIÓDICA) CON IGUAL PERIODO DE CAPITALIZACIÓN}

Para este caso tenemos una tasa nominal vencida conocida, y se requiere hallar una tasa efectiva (periódica), con igual periodo de capitalización, por ejemplo, del 13\% (NMv hallar una tasa efectiva (periódica) mensual.

Para este caso es necesario entender que se va a llevar a una efectiva (periódica) que tiene el mismo periodo de capitalización, para el caso de las nominales se dice que estas son una expresión anual de las tasas periódicas, por consiguiente, solo es necesario multiplicarlas por el número de veces que capitaliza en el año, luego para llevar de una nominal a una efectiva (periódica) solo es necesario realizar la operación inversa, es decir dividirla, quedando de la siguiente manera:

$$
\text { interés }_{\text {efectivo }}=\frac{\text { interés }_{\text {nominal }}}{\text { número de periodos de capitalización }}
$$

Para el caso de estudio, los periodos de capitalización mensual que tiene un año son 12, por tanto, tenemos:

$$
i_{\text {efectivo }}=\frac{13 \%}{12}=1.083 \% \text { mensual }
$$

Para el caso de una nominal vencida a una Efectiva Anual (EA), se puede trabajar con la función financiera en Microsoft Excel, denominada INT. EFECTIVO, como a continuación se muestra:

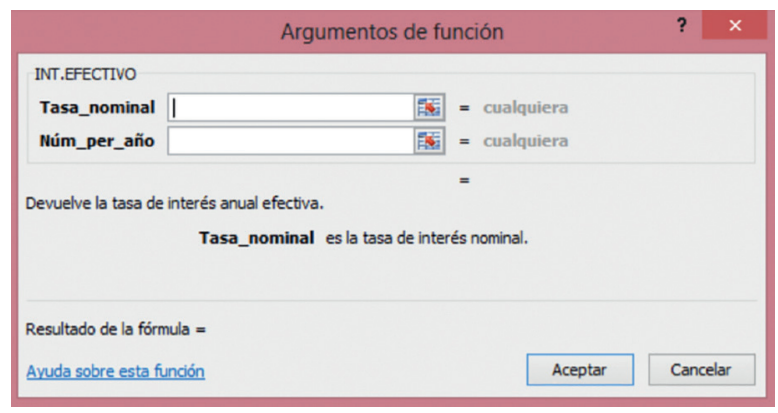

Figura3. Función INT.EfEctivo. Tomado de Microsoft (2016).

Como se puede observar en la figura 2, los argumentos que pide la función son Tasa_nominal y Núm_per_año, el primero es la tasa nominal conocida, y el segundo es la capitalización que tiene la tasa nominal conocida, para el caso de la nominal mensual el número de periodos de capitalización al año es 12, para la trimestral 4, y así sucesivamente.

\section{CASO 5: DE NOMINAL A EFECTIVA (PERIÓDICA) CON DIFERENTE PERIODO DE CAPITALIZACIÓN}

Para este caso tenemos una tasa nominal vencida conocida y se requiere hallar una tasa efectiva (periódica), con diferente periodo de capitalización, por ejemplo, del 13\% (NMV) hallar una tasa efectiva (periódica) trimestral.

Para este caso es necesario entender que se va a llevar a una efectiva (periódica) que tiene diferente periodo de capitalización, para el caso de las nominales se dice que estas son una expresión anual de las tasas periódicas, por 
consiguiente, solo es necesario multiplicarlas por el número de veces que capitaliza en el año, luego para llevar de una nominal a una efectiva (periódica) solo es necesario realizar la operación inversa, es decir dividirla, quedando de la siguiente manera:

$$
\text { interés }_{\text {efectivo }}=\frac{13 \%}{12}=1.083 \% \text { mensual }
$$

Lo anterior demuestra que independiente de la tasa que se requiere hallar, las tasas nominales siempre se dividen por el número de veces que capitalizan, para el caso de estudio se dividió por 12, dado que en un año hay 12 periodos de meses; sin embargo, la tasa que se está requiriendo es trimestral, una vez se tiene periódica solo se cambia el periodo de capitalización, como se muestra en el caso 1.

interés $_{\text {efectivo }}=(1+1.083 \%)\left(\frac{12}{4}\right)-1=3.285 \%$ trimestral

\section{CASO 6: DE PERIÓDICA VENCIDA A PERIÓDICA ANTICIPADA}

Para los casos anteriores trabajamos con tasas vencidas, para este caso, ya surge el concepto de tasa anticipada, para este caso es necesario referirse al grafico 1, en el que se indica el camino para llevar una tasa periódica vencida a una tasa periódica anticipada, de acuerdo con esto, tenemos lo siguiente:

$$
i_{p a}=\frac{i_{p v}}{1+i_{p v}}
$$

Donde;

$i_{p a}=$ Interés periódico anticipado

$i_{p v}=$ Interés periódico vencido

Para este caso se tiene una tasa del 5\% periódica mensual vencida, se requiere una tasa periódica mensual anticipada, para ello tenemos:

$$
i_{p a}=\frac{5 \%}{1+5 \%}=4,762 \% p m a
$$

Para este caso se puede observar que las tasas vencidas suelen ser mayores que las tasas anticipadas, por ello, es necesario conocerla y, saber su manejo, para que permita este conocimiento tomar decisiones acertadas.

\section{CASO ?: DE PERIÓDICA ANTICIPADA A PERIÓDICA VENCIDA}

Haciendo referencia al gráfico 1, donde se indica el camino para llevar una tasa periódica anticipada a una tasa periódica vencida, de acuerdo con esto se tiene lo siguiente:

$$
i_{p v}=\frac{i_{p a}}{1-i_{p a}}
$$

Donde:

$i_{p v}=$ Interés periódico anticipado

$i_{p a}=$ Interés periódico vencido

Para este caso, se tiene una tasa del 5\% periódica mensual vencida anticipada, se requiere una tasa periódica mensual vencida, para ello se tiene:

$$
i_{p v}=\frac{5 \%}{1-5 \%}=5.2653 \%
$$

Como se observa, las tasas periódicas vencidas son siempre superiores a las tasas periódicas anticipadas.

\section{RECOMENDACIONES PARA TRABAJAR LAS TASAS DE INTERÉS}

1. Identifique que tipo de tasa tiene, es decir, si es nominal o es efectiva.

2. Tenga claro cuál es la tasa que quiere encontrar.

3. Luego, ubicar en el grafico 1 el esquema de conversión de tasas y siga la ruta.

4. Confirme si la tasa obtenida es la que buscaba.

Ejemplo: Victoria tiene una necesidad de recursos financieros, ella desea comprar una má- 
quina de coser industrial para su boutique. Solo cuenta con una parte de ese recurso, acude a una institución financiera para que le presten el resto, y le dicen que el crédito que podrían realizarle tiene un interés de 1.9\% mensual, ella quisiera saber cuento le cuesta ese crédito anual:

1. Tipo de tasa: $1.9 \%$ mensual (equivalente a una tasa periódica o una tasa efectiva mensual).

2. Tasa que quiere encontrar: anual, o lo que es lo mismo, Efectiva Anual o EA

3. El esquema nos muestra que debemos aplicar la ecuación:

$$
\begin{gathered}
i_{\text {efectivo }}=(1+i) \frac{n}{m}-1 \\
i_{\text {efectivo }}=(1+1.9 \%) \frac{12}{1}-1=25.34 \%
\end{gathered}
$$

4. Ya Victoria tiene la tasa de interés que necesitaba

\section{¡Atención!}

- De ningún modo multiplique una tasa nominal

- De ningún modo divida una tasa efectiva

- Toda vez que multiplique una tasa el resultado será una nominal

- Toda vez que divida una tasa nominal el resultado será una tasa periódica

- Para llegar a una tasa efectiva siempre debemos partir de una tasa periódica (Espitia y Rojas, 2012). 


\section{TASA REAL O DEFLACTADA}

Como se mencionó al iniciar el texto, hay unas variables económicas que afectan el valor del dinero en el tiempo, una de esas es la inflación ${ }^{4}$, por ello es necesario tenerla en cuenta al momento de determinar cuan rentable es un negocio o una inversión. De acuerdo con Sosa (2006), esta consiste en la diferencia que se presenta cuando se descuentan tasas periódicas o efectivas entres si, o cuando se descuenta la inflación de las tasas de rendimiento totales, se expresa de la siguiente manera:

$$
i_{\text {Real o Deffactado }}=\left(\frac{i_{\text {efectivo }}=\text { interés }_{\text {inflación }}}{=\text { interés }_{\text {inffación }}}\right)
$$

Donde:

$i_{\text {efectivo }}=$ tasa de interés efectiva

$i_{\text {inflación }}=$ tasa de inflación

Por ejemplo, un inversionista tiene una rentabilidad en su negocio del 20\% anual, sin embargo, lo contrata a usted para que por favor le determine el impacto de la inflación sobre su rentabilidad, sabiendo que esta es del 4,44\% anual. Por consiguiente, el impacto que sufriría el inversionista es el siguiente:

$$
i_{\text {Real odeflactado }}=\left(\frac{20 \%-4,44 \%}{1+4,44 \%}\right)=14,9 \%
$$

Como se muestra, el inversionista está teniendo una rentabilidad real o después de inflación del $14,9 \%$ y no del $20 \%$ como inicialmente se pensaba, por consiguiente, es importante entender el impacto de esta variable, al momento de realizar inversiones en cualquier economía.

4 El fenómeno de la inflación se define como un aumento sustancial, persistente y sostenido del nivel general de precios a través del tiempo (Banrep, 2017). 


\section{Apartado finAL}

Al concluir esta nota de clase, el estudiante deberá haber desarrollado la capacidad de conocer las diferentes tasas de interés que se dan en el mercado colombiano, las formas en que se trabajan, pero además, deberá conocer la forma en que se utilizan para tomar decisiones, también, tener clara la importancia de identificar que el dinero a través del tiempo va tomando valores diferentes, además de las variables que lo afectan, esto nos ayudará a comprender por qué el dinero que utilizamos no es gratis, por consiguiente, la importancia de ser eficientes en el manejo en su manejo.

\section{Consideración final}

El alto analfabetismo financiero de nuestra sociedad, acompañado del poco fomento que se le hace al tema financiero en las instituciones educativas, llámese nivel básico, medio y superior, inciden en que las personas tomen decisiones, sin tener en cuenta algunos principios básicos como el costo del dinero, situación que lleva a que los ciudadanos utilicen los instrumentos financieros, asumiendo costos bastaste excesivos, incluso muchas veces innecesarios. Por tanto, es necesario que desde el sector académico se creen y fomenten estrategias que permitan disminuir estas brechas educativas, que permitan que los ciudadanos tomen decisiones no llevadas por la emoción sino por la razón, en este sentido, el documento se desarrolló para que quien lo lea y trabaje, pueda adquirir unas competencias mínimas necesarias y suficientes, que le permitan tomar decisiones asertivas no basadas en la emoción, si no sustentadas y fundadas en elementos prácticos. 


\section{EJERCICIOS PARA PRACTICAR}

1. Dada una tasa del $10 \%$ cuatrimestral, hallar una tasa semestral.

\section{R. $15,3690 \%$}

2. Dada una tasa del $2 \%$ mensual, hallar una tasa EA.

\section{R. $26,82 \%$}

3. Dada una tasa del 15\% NTV, hallar una tasa efectiva trimestral.

\section{R. $3,75 \%$}

4. Dada una tasa del 10\% EA, hallar una tasa anual con capitalización semestral.

\section{R. $9,7618 \%$}

5. Dada una tasa del 15\% Nsv, hallar una tasa trimestral.

\section{R. $3,6822 \%$}

6. Dada una tasa del 18\% NBV, hallar una tasa anual con capitalización mensual.

\section{R. $17,8670 \%$}

7. Dada una tasa del $14 \%$ NTV, hallar una tasa EA.

\section{R. $14,7523 \%$}

8. Dada una tasa del 3\% efectiva mensual, hallar una tasa periódica mensual anticipada.

\section{R. $2,9126 \%$}

9. Dada una tasa del $4 \%$ periódica trimestral anticipada, hallar una tasa semestral vencida.

\section{R. $8,5069 \%$}

10. Dada una tasa del $10 \%$ EA, hallar una tasa NTA.

\section{R. $9,4184 \%$}

11. Dado una tasa del $15 \%$ anual semestre vencido, hallar una tasa NSA.

\section{R. $13,9534 \%$}

12. Matías tiene 3 alternativas para invertir, y lo contrata a usted para que lo asesore cual alternativa escoger.
a. $10 \% \mathrm{EA}$
b. $3 \%$ efectiva cuatrimestral
c. $9 \% \mathrm{NMV}$

\section{R. La mejor alternativa es la a}

13. Edder espera financiar su proyecto, para ello ha consultado 3 instituciones bancarias que le han otorgado los siguientes costos de financiación:
a. El banco A otorga una tasa del 15\% NSA
b. El banco B otorga una tasa del 15\% NBA
c. El banco C otorga una tasa del $15 \%$ NTA

14. Dada una tasa del $12 \%$ EA, hallar una tasa:
a. NBV
e. NSA
b. NBA
f. NSV
C. NTV
g. NMV
d. NTA
h. NMA

15. Dada una tasa del $15 \%$ anual con capitalización mensual, hallar una tasa:
a. Mensual
e. Periódica semes- tral anticipada
b. Trimestral
f. Periódica trimes- tral anticipada
c. Semestral
g. Periódica cuatri- mestral antici- pada
d. Periódica
h. EA anticipado 
16. Consulte la página web del Banco de la República ${ }^{5}$ y verifique la inflación anual y determine la tasa real para las siguientes rentabilidades de un inversionista:
a. $15 \%$
b. $12 \%$
c. $35 \%$

17. Si el inversionista requiere una rentabilidad real del 18\% ¿cuál es la rentabilidad mínima que debe darle sus inversiones? teniendo en cuenta la inflación consultada en el punto anterior.

18. Teniendo en cuenta el punto 16 y 17, identifique cuál es la tasa de inflación máxima que está dispuesto a tolerar, para cada uno de los casos del punto 16 .

19. Doña Gladis necesita un dinero para comprar su vivienda, ella le pregunta a usted, cuál de las opciones que le ofrece su banco amigo debe elegir, las alternativas son las siguientes:
a. $35 \%$ nominal anual capitalizable se- mestralmente
b. $38 \%$ nominal anual capitalizable tri- mestralmente
c. $32 \%$ nominal semestral capitalizable mensualmente

20. Hallar la tasa efectiva anual equivalente a:
a. $5 \%$ efectivo mensual
b. $8 \%$ efectivo trimestral
c. $12 \%$ efectivo cuatrimestral
d. $6 \%$ efectivo bimestral

21. Hallar la tasa efectiva mensual equivalente a:
a. $1 \%$ efectivo quincenal
b. $5 \%$ efectivo semestral
c. $12 \%$ efectivo anual
d. $0.05 \%$ diario

22. Hallar la tasa de interés EA equivalente a:
a. $25 \%$ capitalizable cuatrimestralmente
b. $25 \%$ capitalizable mensualmente
c. $25 \%$ capitalizable bimestralmente

23. Teniendo en cuenta el punto 22, determinar cuál es la opción que más conviene a una persona que va a realizar una inversión y se le presentan estas opciones. 


\section{REFERENCIAS}

ASOBANCARIA. (2015). Programa de educación financiara de los bancos en Colombia. Recuperado de http://www.asobancaria.com/sabermassermas/tasas-de-interes/

Banco de la República. (2017). ¿Qué es la inflación? Recuperado de http://www.banrep.gov.co/es/contenidos/ page/qu-inflaci-n

Espitia, R. y Rojas, G. (2012). Manual didactico de matemáticas financieras. Bogotá: Universidad EAN.

García, J. (2008). Matemáticas financieras con ecuaciones de diferencias finitas. Bogotá: Pearson Prentice Hall.

Gomero, H. (2006). Fundamentos tecnicos de la matematica financiera. Lima: Fondo Editorial Universidad Pontificia Catolica del Perú.

Meza, J. (2008). Matemáticas financieras aplicadas, uso de la calculadora financiera y Excel. Bogotá: Eco Ediciones. Microsoft. (2016). Excel.

Ramírez, J. y Martínez, E. (2010). Matemáticas financieras interés, tasas y equivalencias. Bogotá: Editorial Trillas. Sabogal, J., Cayón, E. y Rivera, J. (2007). Matematicas financieras en Microsoft Excel. Bogotá: Editorial Pontificia Universidad Javeriana.

Sosa, R. (2006). Manual de ingeniería económica. Santa Marta: Fondo Editorial Universidad de Eafit.

Soto, E. y Serna, C. (2015). La contabilidad en función de la sustentabilidad: una mirada desde el desarrollo económico alternativo. Quipukamayoc, 23(44), 109-118. 


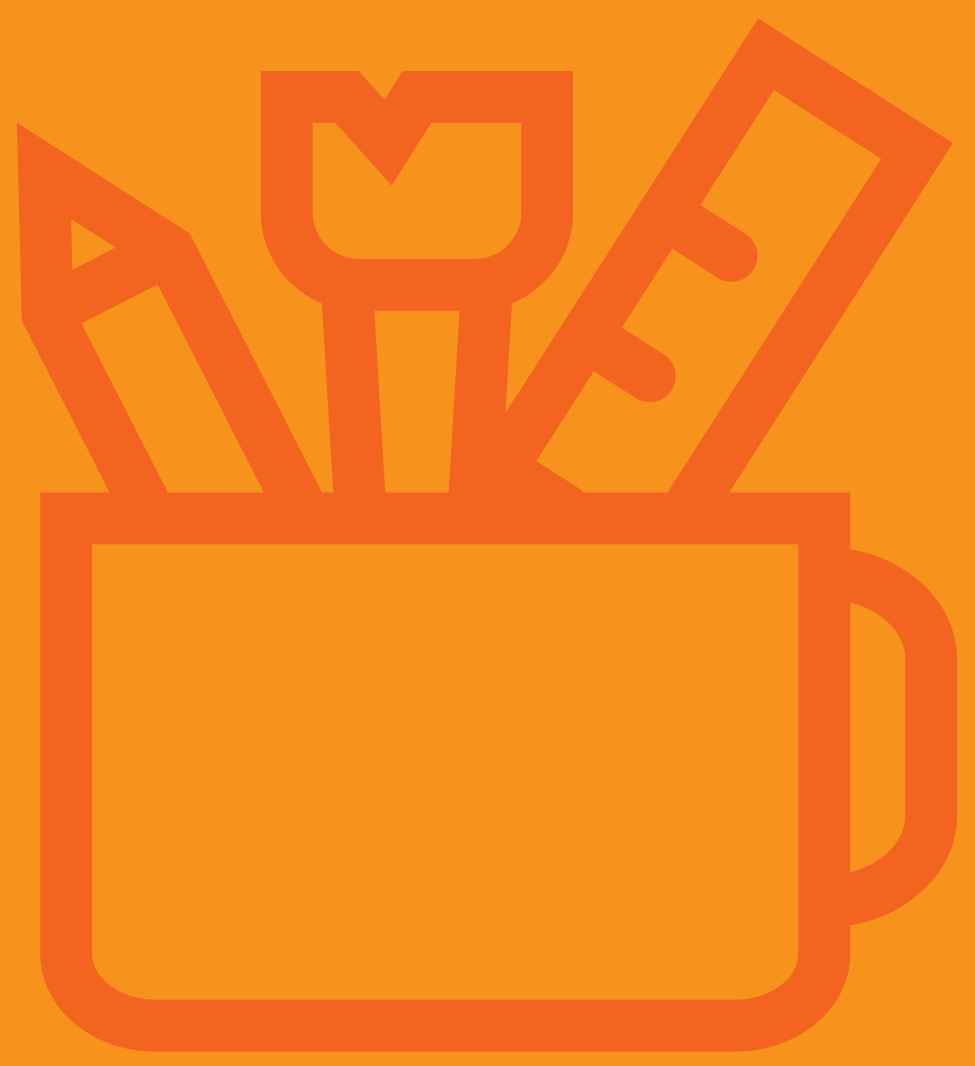

